\title{
R-Gada: a fast and flexible pipeline for copy number analysis in association studies
}

Roger Pique-Regi ${ }^{1,2^{*}}$, Alejandro Cáceres ${ }^{3,4}$, Juan R González $3,4,5^{*}$

\begin{abstract}
Background: Genome-wide association studies (GWAS) using Copy Number Variation (CNV) are becoming a central focus of genetic research. CNVs have successfully provided target genome regions for some disease conditions where simple genetic variation (i.e., SNPs) has previously failed to provide a clear association.

Results: Here we present a new R package, that integrates: (i) data import from most common formats of Affymetrix, Illumina and aCGH arrays; (ii) a fast and accurate segmentation algorithm to call CNVs based on Genome Alteration Detection Analysis (GADA); and (iii) functions for displaying and exporting the Copy Number calls, identification of recurrent CNVs, multivariate analysis of population structure, and tools for performing association studies. Using a large dataset containing 270 HapMap individuals (Affymetrix Human SNP Array 6.0 Sample Dataset) we demonstrate a flexible pipeline implemented with the package. It requires less than one minute per sample (3 million probe arrays) on a single core computer, and provides a flexible parallelization for very large datasets. Case-control data were generated from the HapMap dataset to demonstrate a GWAS analysis.

Conclusions: The package provides the tools for creating a complete integrated pipeline from data normalization to statistical association. It can effciently handle a massive volume of data consisting of millions of genetic markers and hundreds or thousands of samples with very accurate results.
\end{abstract}

\section{Background}

High resolution oligonucleotide array platforms with millions of markers have enabled the study of copy number variation $(\mathrm{CNV})$. CNVs are alterations of the genome in which small segments of DNA sequence are duplicated (gained) or deleted (lost) [1-5]. These alterations can affect regulatory regions or coding portions of a gene, and have been found associated with a number of genetic disorders and some complex heritable diseases [6].

In contrast to SNPs, which rely on having linkage disequilibrium with the underlying causal mutation, $\mathrm{CNVs}$ are more likely to point the underlying biological cause that affects the phenotype of interest. This is because the duplication or deletion can readily explain a gain or loss in gene expression levels. While it has been shown that that common CNVs can be tagged well with

\footnotetext{
*Correspondence: rpique@ieee.org; jrgonzalez@creal.cat

'Signal and Image Processing Institute, Viterbi School of Engineering,

University of Southern California, EEB 400, 3740 McClintock Ave, Los Angeles, CA 90089-2564, USA

${ }^{3}$ Center for Research in Environmental Epidemiology (CREAL), Doctor Aiguader, 88, Barcelona, 08003, Spain
}

SNPs (77\%) [4,5], Conrad et al. [5] also argue for the need to consider all classes of variation (SNPs and all structural variants, common and rare) in genome wide association studies. In this context, CNVs affecting small regions (in the order of a few kilobases) or structurally complex CNVs (e.g., CNVs without shared boundaries, or nested CNVs) as seen by [7] require high resolution microarrays (millions of probes) and very accurate techniques in placing the copy number change locations. Detection of rare CNVs also requires large sample sizes making essential computationally efficient tools for the CNV extraction.

The complete analysis of $\mathrm{CNV}$ association requires three main steps: i) normalization, ii) segmentation, and iii) common alteration and association analysis. The objective of the normalization is to clean as much as possible the data from all known sources of experimental variation unrelated to copy number changes. In contrast to SNP association studies, where we can perform genotyping for each probe individually after normalization, studies using CNVs require the identification of contiguous stretches of probes with the same underlying 
copy number change. Thus, CNV association studies require a more complex pipeline. In this paper we present a new $R$ package (R-GADA) that facilitates the implementation of a complete pipeline from data normalization to the final CNV association analysis. Since data normalization is highly specific of each microarray technology the package can import normalized data from several packages such as aroma. affymetrix and tools provided by Illumina and Affymetrix. The segmentation algorithm implemented in the package is based on the Genome alteration detection analysis (GADA) [8]. Compared with circular binary segmentation (CBS) [9], one of the most accurate segmentation algorithms available, GADA has similar or better accuracy and is several orders of magnitude faster. Recently, GADA has been used by [5] and [10] to analyze very large data sets. GADA had a limited availability as a $\mathrm{C} /$ Matlab library, and lacked a complete pipeline to facilitate the analysis that the presented package provides. The package presents new functionality for automatically splitting the data in files for each sample and chromosome, which can then be analyzed in parallel in a multicore computer. After fitting the GADA model, RGADA offers efficient methods for adjusting the final segmentation sensitivity and false positive rate, as well as a complete set of tools for visualizing and reporting copy number alterations. Most prominently, R-GADA offers tools for identifying population structure in the $\mathrm{CNV}$ data that can be taken into account on the final case-control CNV association study. The pipeline offers a flexible computational framework that can easily accommodate changes in any stage of the analysis to fulfill the requirements of the study.

The package is demonstrated on a large dataset consisting of 270 Hapmap samples (Affymetrix Human SNP 6.0 array sample data). We generated case-control data associated with some CNVs illustrating a potential scenario encountered on GWAS using CNVs. The latter exemplifies the entire analysis process from data normalization, segmentation, recurrent CNV analysis, population structure correction, to the final statistical association analysis; see the illustration of pipeline analysis in Figure 1. More examples are provided through the R-GADA user manual and the Google group http:// groups.google.com/group/gadaproject used for providing support.

\section{Software main features}

\section{Importing normalized data to gada}

Data can be imported to gada from Illumina, Affymetrix or any other platform that provides information about ratio intensities such as aCGH. We have implemented three different functions for this purpose: setupGADAIllumina. setupGADAaffy and setupGADAgeneral.

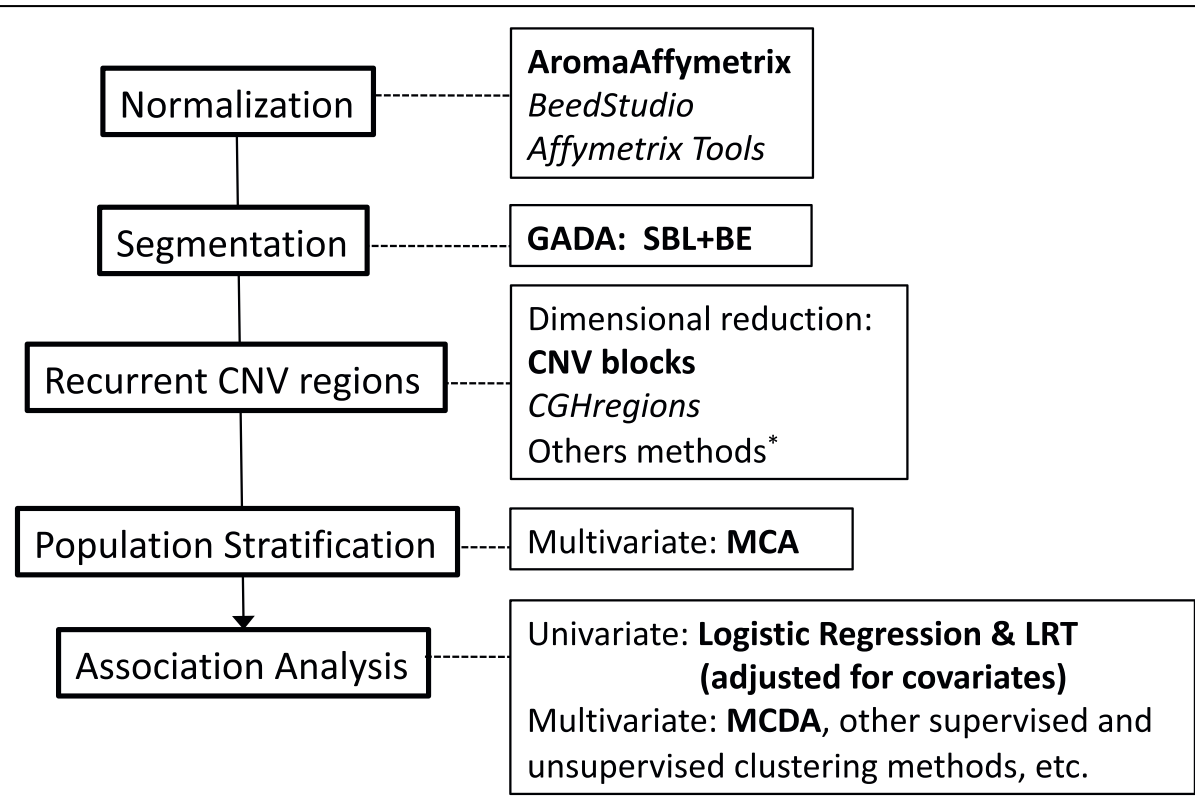

*see Rueda and Diaz-Uriarte (2008)

Figure 1 Pipeline analysis. Analysis strategies for CNV association studies. On the left, we show the main steps of the pipeline, and on right the algorithms used in each step. The algorithms in bold are explicitly used in the present paper; alternatives procedures, easily adaptable in the pipeline, are also shown. 
setupGADAIllumina function uses information obtained from BeadStudio tools http://www.illumina.com. The software allows information to be provided in either a unique text file or a file per individual (or groups of individuals). Alternatively, setupGADAaffy can import data from the Affymetrix Genotyping Console 3.0 (GTC3) http://www.affymetrix.com that extracts normalized $\log _{2}$ ratio intensities from a collection of CEL files. The gada package also handles files containing $\log _{2}$ ratios of other platforms, such as aCGH with the function setupGADAgeneral, which can also be used for cases without chromosome position information. Throughout this paper we interchangeably use the terms $\log _{2}$ ratios or hybridization intensity ratios when we refer to these normalized values.

\section{Breakpoint identification using sparse Bayesian learning (SBL)}

The underlying model for the DNA copy number and the hybridization ratio intensities observed for each probe in the array is illustrated in Figure 2. The number of copies for each autosomal portion of the human genome is generally 2 but, sometimes, small portions of the DNA are duplicated or deleted originating CNV polymorphisms.

The $\log _{2}$ ratio of the array probes increases with the number of times its underlying DNA sequence is present in the genome. The normalization step, which is specific of each array platform, should correct for inherent array biases; so contiguous probes sampling the same CNV have the same $\log _{2}$ ratio average.

Thus, the problem of copy number detection can be casted as a segmentation analysis, where the objective is to identify the set of breakpoints and copy number values that most likely originated the observed data. In the GADA model, the experimental data $y$ is explained by a copy number signal $x$, made of piece wise constant segments, and a random error $\epsilon$,

$$
y=x+\epsilon=\mathbf{F w}+\epsilon
$$

The piece-wise component can be conveniently decomposed on a special base $\mathrm{F}=\left\{f_{m}\right\}_{m}=0 . . M-1$ of step functions at probe $m$. If $x$ has very few breakpoints, then most of the coefficients of the expansion will be zero, and thus the vector of weights $\mathbf{w}$ will be sparse.

Using sparse Bayesian learning (SBL) [11], this sparse vector of weights can be computed as a maximum a posteriori estimate

$$
\hat{\boldsymbol{w}}=\arg \min _{w}-\log p(y \mid \mathbf{w})-\log p(\mathbf{w} \mid \alpha)-\log p(\alpha),
$$

where the first two terms are normally distributed - $N$ $(\boldsymbol{F} \boldsymbol{w}, \sigma 1)$ and $\Pi_{m} N\left(w_{m} \mid 0, \alpha_{m}^{-1}\right)$ respectively -and the vector of hyperparameters $\alpha$ follows a gamma distribution $\Pi_{m} \Gamma\left(\alpha_{m} \mid a, b\right)$. While $\alpha$ and other distribution

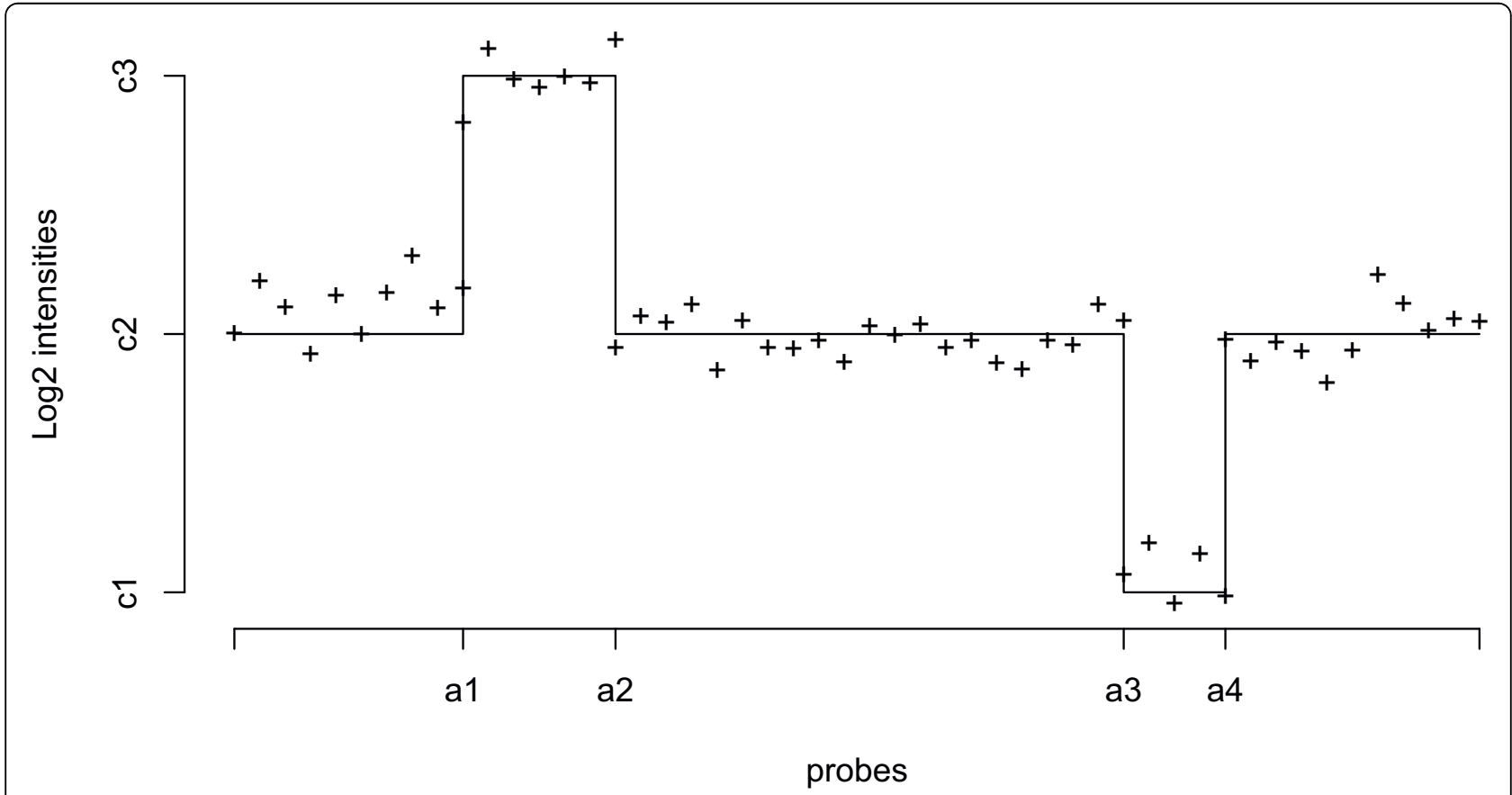

Figure 2 Probe $\log _{2}$ ratio intensities. Schematic representation of probe $\log _{2}$ ratio intensities with two underlying CNVs. Four breakpoints (a1, $a 2, a 3$ and $a 4$ ) separate two altered segments with average intensities $c 3$ and $c 1$, from the unaltered segments with baseline $c 2$. 
parameters are estimated from the data, the parameters $a$ and $b$ are directly controlled by the user.

Typically $b$ is set to zero as an uninformative prior, and $a$ takes values within the range 0.2 and 0.8 . The user then adjusts the level of sparseness solely with the parameter $a$. The reader is referred to Pique-Regi et al. [8] for more details on the application of Sparse Bayesian Learning (SBL) to segmentation analysis.

\section{Backward elimination (BE)}

The significance of each segment's breakpoint is assessed with the $t$-statistic [8], computed with the parameter estimates provided by the SBL step.

$$
t_{j}=\frac{|\hat{w} j|}{\sqrt{\sigma^{2}\left[F_{I}^{\prime} F_{I}\right]^{-1}}} .
$$

where $F_{I}$ is the matrix representation of the reduced basis $F$ for which $w_{j} \neq 0$. Breakpoints with small $t$ are discarded with a backward elimination (BE) procedure, until the segment with lowest $t$ achieves a predefined threshold $T$. This backward elimination ranking of breakpoints with the adjustment of $\mathrm{T}$ is obtained with a very small computational cost.

The SBL and BE procedures make no assumptions on the amplitude of the reconstructed segments. The objective is to provide a nearly optimal set of amplitudes and breakpoint positions that best fits the $\log _{2}$ ratios observed in the array. Once the breakpoints are fixed, in order to achieve the minimal residual error, the amplitude corresponding to each segment is given by the average hybridization level of all the probes that fall inside that segment. The consequence of this model, is that segments that correspond to the same underlying copy number state may be given a different reconstruction amplitude; and, an additional step has to be used to classify these segments into a copy number $(0,1,2$, $3,4, \ldots)$ or alteration status (Non-Altered, Gain and Loss). Here, we adopt the latter three state classification strategy and focus on alterations not comprising the extent of a whole chromosome. Hence, we first estimate the base-line Non-Altered amplitude by calculating the median value of all reconstructed segment amplitudes in a chromosome. Then, we use the same threshold $\mathrm{T}$ to classify all the segments into Gain (Loss) state if the segment amplitude is significantly above (below) the base-line amplitude, or into Non-altered state otherwise. This approach classifies the relative copy number state of each segment with respect the number of copies the chromosome has. For cases, where whole chromosome alterations are expected, the base-line amplitude can be fixed by the user.

\section{Multivariate analysis}

The copy number calls generated in the previous section are categorical variables with three levels: loss, no-change and gain in copy number. For this data, Multiple Correspondence Analysis (MCA) [12] can be used as a unsupervised group classification. One of features of MCA is its principle of distributional equivalence, which assures invariance in the results when rows or columns with identical conditional proportions are merged. This allows the identification of common CNV regions that can be used in the analysis without loss of information. Note that this feature is not required in SNP analysis that use PCA. A supervised discrimination of $\mathrm{CNV}$ maps can also be performed with a Multiple Correspondence Discriminant Analysis (MCDA). In this case, CNVs that are important for the discrimination can be ranked according to their correlation to the class direction $(\lambda)$ on the $k$-th principal axis subspace [13]

$$
\rho_{j}(\lambda)=\frac{\sum_{i=1}^{l} V_{i}(\lambda)\left(x_{i j}-x_{j}\right)}{\sqrt{\sum_{i=1}^{l} V_{i}(\lambda)^{2} \sum_{i=1}^{l}\left(x_{i j}-x_{j}\right)^{2}}},
$$

rather than to the axes themselves. In this equation $V_{i}$ $(k)$ is the coordinate of class centroid $i$ in the direction of class $\lambda ; x_{i j}$ is the aggregated indicator matrix of segment callings for the group $i$ and segment $j$. This correlation can be tested for significance with a permutation test on the class labels and can also be used to rank the variables according to their importance to discriminate each class. The ranking allows the selection of a group of CNVs which are most relevant for the discrimination. A subsequent unsupervised classification with the selected variables confirms their relevance in population labeling.

\section{Implementation}

The package streamlines CNV segmentation analysis, separated into three major processes: 1) Importing data files containing the normalized $\log _{2}$ ratio intensities of either Illumina or Affymetrix arrays or any other technology such aCGH, 2) applying GADA and 3) summarizing and visualizing results. These three steps can be performed for an individual array or for multiple samples. In the latter case, the software separates data into different files, one for each sample, allowing an easy way to perform parallel computation.

The functions of the package, their call and arguments are illustrated in detail on the Additional File 1 where specific example applications are described. The file is the user's manual that gives a detailed tutorial for analyzing specific data-sets. 


\section{Importing normalized data}

The pipeline analysis of several samples requires a special data management for the input data. Specialized functions like splitDataBeadStudio, for Illumina data, and those in aroma.affimatrix package are used to build the directory structure required by gada. Raw data for $n$ subjects stored in a single file is split into $n$ files within the subdirectory "rawData", created automatically in the working directory. Loading data into the $\mathrm{R}$ session is performed by either setupParGADAIllumina or setupParGADAAffy. An R object of type "setupGADA" is created and taken as input for the segmentation routines. This data handling ensures smooth conversion of array output to suitable input for the segmentation algorithms.

\section{Segmentation procedure}

Segmentation is made of two consecutive algorithms implemented in two separate R-functions within the gada package. The first function (parSBL) uses sparse Bayesian learning (SBL) to discover the most likely positions and magnitudes for a change in copy number, i.e. the breakpoints. The SBL model is governed by a hierarchical Bayesian prior, which is uninformative with respect to the location and magnitude of the copy number changes but restricts the total number of breakpoints. Sensitivity, given by the maximum breakpoint sparseness, is controlled by the hyperparameter $a$. The second function (parBE) is an algorithm that uses a backward elimination (BE) strategy to rank the statistical significance of each breakpoint obtained from SBL. The results from parSBL and parBE are stored in separate files, one for each sample.

\section{Parallel computation}

The package enforces a strict directory structure on the working directory to perform the analysis of multiple samples. However, the only required directory is that containing the raw data. This structure is designed to reduce memory demands and to easily set up simultaneous parallel processes, a main and novel feature of the present implementation.

While the analysis is readily installed to perform single background process, if multiple processors are available, the computing time can be greatly reduced. This facility is implemented with the snow and Rmpi packages http://cran.r-project.org/web/packages/snow. Parallel computation is straight-forward after loading the previous packages.

\section{Single array}

In the case of analyzing a single sample, the file management system is not required. Data can be directly loaded on the $\mathrm{R}$ console from a text file containing the $\log _{2}$ ratios for each probe. Setting up the loaded data into a "setupGADA" object is still required and depends on its original format. This is done with setupGADAIllumina, setupGADAAffy or setupGADAgeneral as previously described. Segmentation follows from the sequential application of the single array functions SBL and BackwardElimination.

\section{False discovery rate}

The critical value $T$ required in the BE step is used to establish the final degree of desired sparseness by adjusting the level of FDR. The procedure also requires the specification of the parameter MinSegLen, which determines the minimum number of altered probes in each segment. The two-step strategy (SBL and BE) allows the flexible adjustment of sparseness after breakpoint estimation. Specifically, optimal $T$ is found without re-computation of parSBL. Table 1 shows suitable combinations of parameters to achieve desired sensitivity and FDR.

A simulation study illustrates the behavior of FDR as function of $T$ and MinsegLen. The general simulation model follows [14], however, we have changed the length of the altered and non-altered segments for more realistic matching of non-cancer settings. Figure 3 shows the FDR for different $T$ and MinSegLen values. As expected, FDR decreases when $T$ or MinSegLen increases.

\section{Summarizing and displaying segmentation results}

The segmentation obtained by GADA returns a collection of segments with breakpoint positions on the most likely locations for copy number change, and segment amplitudes corresponding to the average $\log _{2}$ ratios of the probes falling between two consecutive breakpoints. The summary function determines which of these ratios correspond to possible changes in copy number, by establishing alteration boundaries. The limits, if not selected by the user, are estimated using the $\times$ chromosome of a reference population containing males and females. Segments with amplitudes below (above) the interval limit are reported with a loss (gain) in copy number. All other segments are considered as nonaltered. The result of summary is an R-object of class data.frame that stores the name, chromosome and position of the initial probe of each altered segment, and the number of subject with such alteration.

\section{Table 1 Parameter selection}

\begin{tabular}{ccc}
\hline (higher sensitivity, higher FDR) & $<->$ & $\left(a_{\alpha}=0.2, T>3\right)$ \\
& $<->$ & $\left(a_{\alpha}=0.5, T>4\right)$ \\
(lower sensitivity, lower FDR) & $<->$ & $\left(a_{\alpha}=0.8, T>5\right)$ \\
\hline
\end{tabular}

Recommended settings of $a$ and $T$ depending on the desired level of sensitivity and FDR. 


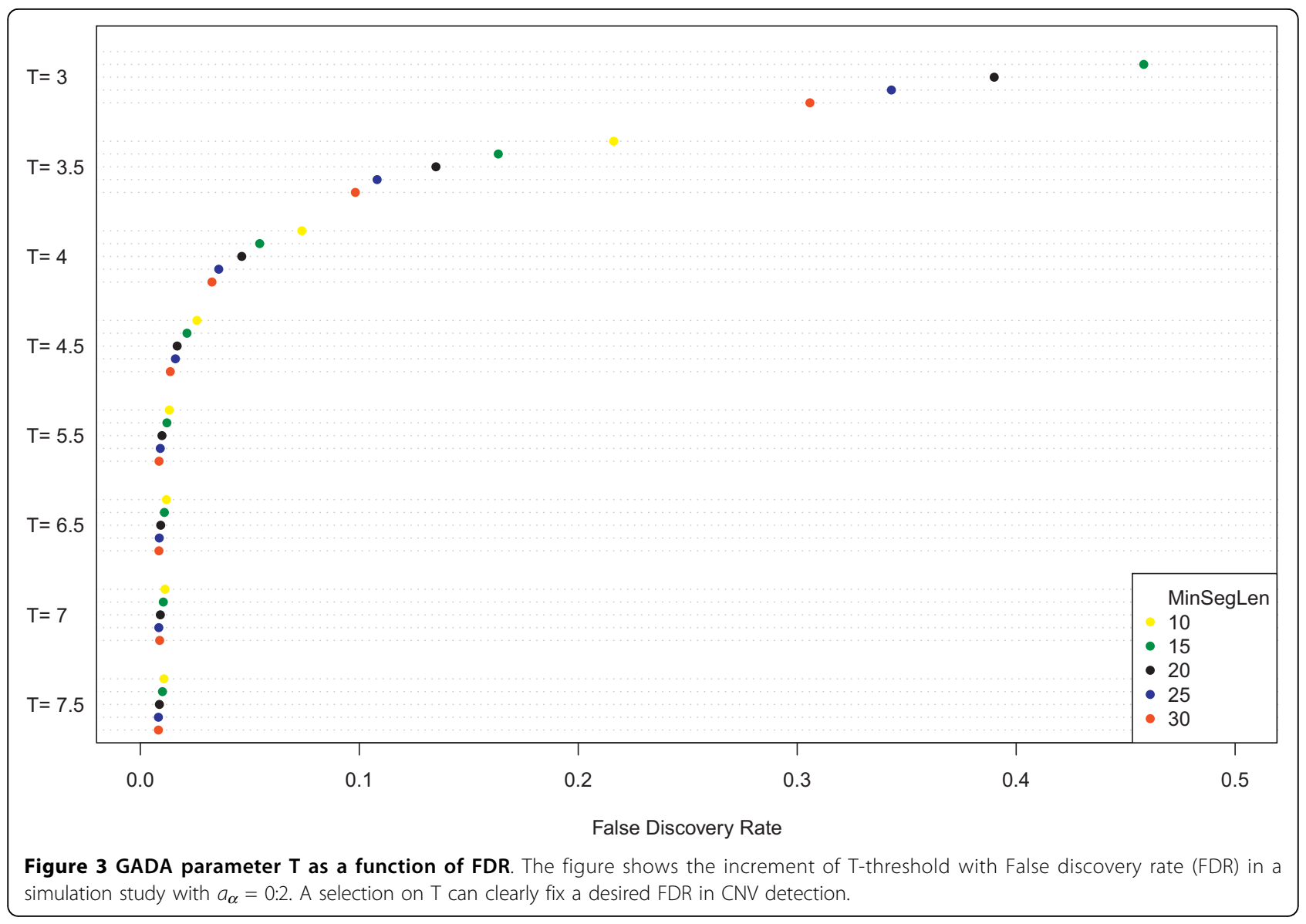

The $\mathrm{R}$ package plotrix is used to display the resulting segments within a given chromosome or across the whole genome. As a data. frame, the output is ready for downstream association analysis implemented on $\mathrm{R}$. Alternatively, they can be exported in BED format and imported into any of the popular genome browsers such as those from UCSC and Ensembl.

\section{Multivariate Analysis}

The output of the GADA algorithm is easily converted to a matrix of CNVs(columns) and subjects (row) with entries -1 (loss), 0 (no-change) and 1(gain). The function getReducedData produces the matrix for all the CNVs across the genome; it also groups and filters the variables. Contiguous variables are grouped together within blocks whose extremes do not differ in more than VarSimil\%, and variables (or blocks) with less than 1-subVariation: across subjects are discarded. The parameters are set by the user. If desired, this data reduction can also be performed with other algorithms (i.e. CGHregions), which can be, nevertheless, slower and less flexible for dealing with a large amount of data.

MCA is performed with the general function dudi. acm from ade4, an $\mathrm{R}$ package for multivariate analysis.
The variable scores, onto the principal component sub-space, can be used as co-variates of an association test to account for population stratification. Specific plots are implemented for displaying such analyses. For identifying a subset of CNVs with the highest prediction power, an MCDA can be performed. More specialized functions, enveloping those of ade4, have been developed for this type of analysis. This functions include discrimin. cnv and rank.variables. The second of these ranks the CNVs according to their correlation onto the population centroids. Variables can be selected by establishing a minimum value for the correlation, assessing their statistical significance with a permutation test, or maximizing the cross-validation accuracy on a train set. Note that the two last options are more computational demanding.

\section{Association Analysis}

Association analysis is implemented for a logistic regression model and a likelihood ratio test (LRT). The function multiCNVassoc repeatedly computes the LRT, comparing a model that includes covariates (e.g., sex, age, population, etc.) with the same model for which the CNV under consideration has been added. The p-value is then computed using a Chi-squared test. This 
function returns an object of class "multiCNVassoc" that can be printed or plotted using the $\mathrm{R}$ generic functions print and plot respectively. We also provide a procedure to deal with multiple comparisons. The function get $\mathrm{PvalBH}$ returns the p-values for association corrected by the Benjamini-Hochberg $(\mathrm{BH})$ method. This is a heuristic method that is robust against positive dependence and increasingly conservative as correlation increases. Association tests that need to be corrected for population stratification can incorporate as covariates the scores of the first eigen-values of an MCA.

Note that in this association study CNVs are not treated as simple SNPs. One single probe (not all the probes falling under a $\mathrm{CNV}$ ) is chosen as a representative of a CNV block. This forms a reduced matrix that is obtained as explained in the previous section. If a complex structure of overlapping CNV segments exists across the samples, multiple alternative segments will be considered for association. Although, the example considered here only covers common CNVs, we anticipate that the approach presented here will be helpful for analyzing more complex scenarios and for developing better association tools. Particularly for rare CNVs, novel association analysis methods are necessary where genes (or DNA regulatory element) are disrupted at different non-overlapping parts.

\section{Connection with Aroma.Affymetrix}

GADA can be called within the Aroma. Affymetrix package http://www.aroma-project.org/, which provides a comprehensive normalization strategy, as used by [6] and an analysis framework for copy number detection and visualization. GADA segmentation tools are specially adapted to the package pipeline, by replacing, within an Aroma.Affymetrix session, the function CbsModel() by our implemented function GadaModel().

\section{Results and Discussion}

In this section we show the results obtained in the main stages of a typical analysis pipeline (the main features of the modules and the type of input/output information they use). The results are illustrated with two sample data sets. The first consist of a set of 8 samples using Illumina Human1M-Duo arrays with $3 \mathrm{M}$ probes. The second dataset consist of 270 lymphoblastoid cell-lines from the HapMap project using Affymetrix SNP 6.0 arrays. A more detailed, step by step, description of these analyses can be followed in Additional file 1 .

\section{Segmentations}

The sample data for eight (non HapMap) subjects has over $3 \mathrm{M}$ probes and comes in a BeadStudio format. The first three columns contain name of probe, chromosome and genomic position. Subsequent columns store the $\left(\log _{2}\right)$ ratios for each individual. After splitting into files, loading and setting up, the data was segmented with an $a=0.8$ and $T=8$, a choice to optimize FDR. A summary of the output of such segmentation is illustrated as follows:

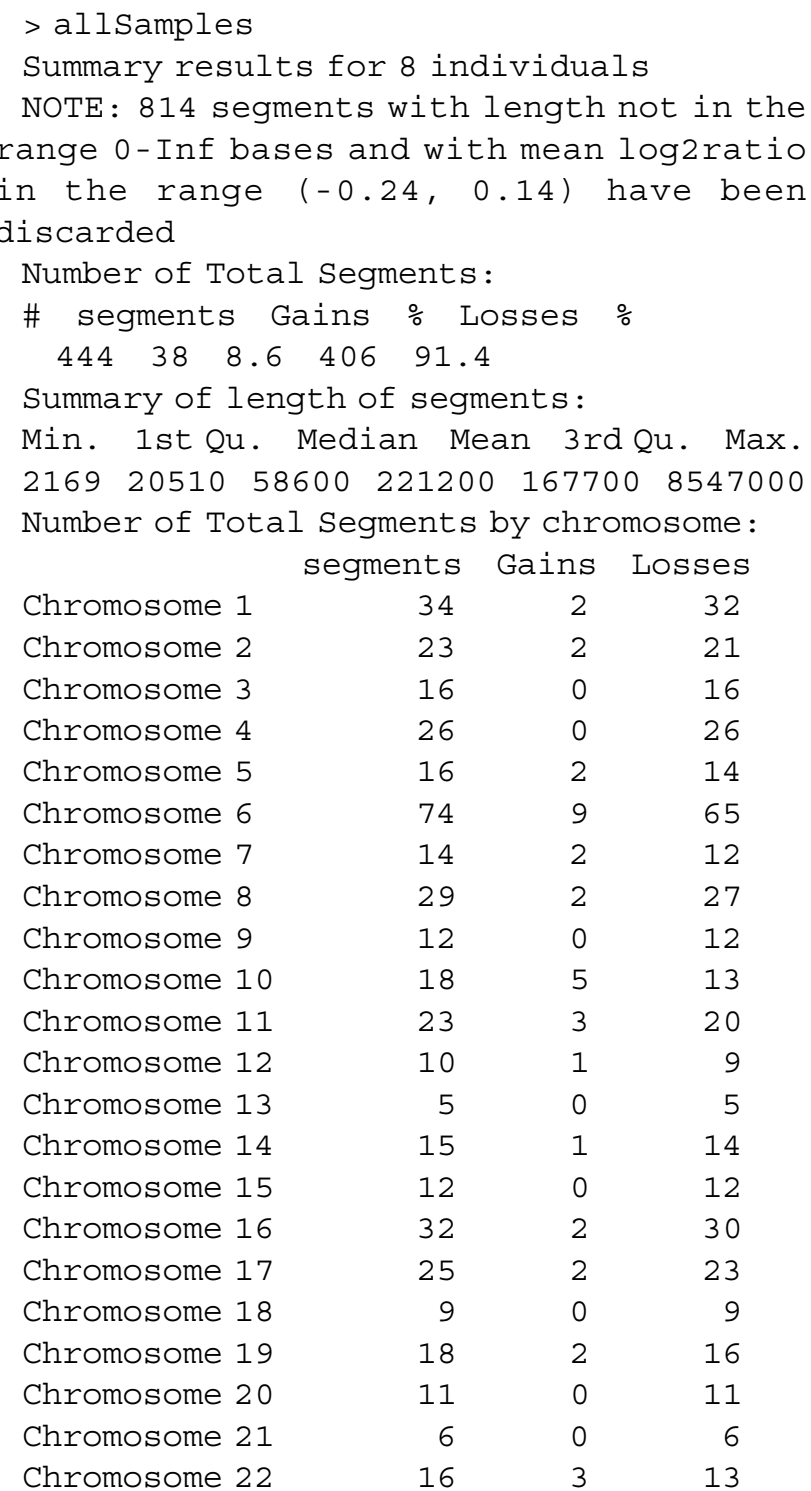

In this example the limits of the non-altered probes were estimated at $(-0.24,0.14)$ and all segments are considered (length: 0-Inf). If the user is interested in considering only segments in a given range, the argument length. base should be changed indicating their minimum and maximum size. Plots of the segments are readily obtained for the whole genome (Figure 4) or a given chromosome (Figure 5). The processing time for the whole analysis (Mac OS $\times 10.4 .112 .33 \mathrm{GHz}$ ), set up sequentially (not parallel), for eight subjects was approximately $7 \mathrm{~min}$ 30 s. Specifically: loading and setting up data: 200.3 s, SBL: $180.1 \mathrm{~s}$ and BE: $63.9 \mathrm{~s}$. These times are reduced proportionally to the number of computing cores if the process is 


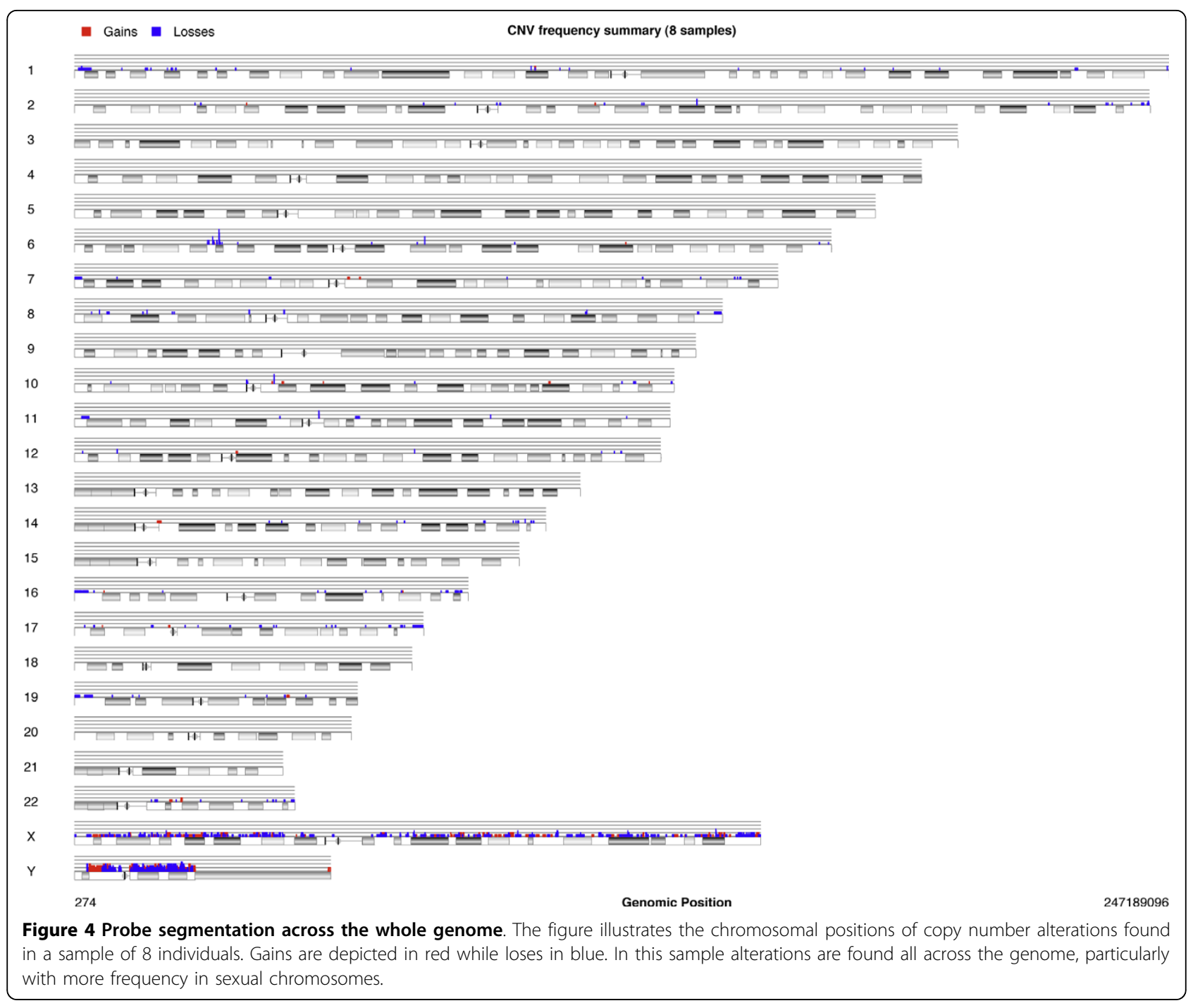

parallelized. A previous study [8] showed that the differences in sensitivity and FDR between GADA and CBS are small $(<3 \%)$. However, GADA is 100 -fold faster. Here we show that a complete implementation of the algorithm with functions for data handling, segmentation and output writing is still computationally efficient. Given that the core of the segmentation is only two sequential functions, the method is amenable for incorporation as a routine step into association analysis.

The gain/loss ratio of $8.6 \%$ versus $91.4 \%$ obtained on this 8 samples is different of that of HapMap samples. For the HapMap samples, we obtain $26.2 \%$ gain and $73.8 \%$ loss CNV segments which is similar to that previously reported by [5]. This higher number of deletions than duplications may be attributed to the greater technical challenge of robustly detecting duplications using oligonucleotide arrays.

\section{Multivariate analysis}

MCA analysis was performed for the HapMap http:// www.hapmap.org sample population of 270 subjects (30 trios of CEU and Yoruban each, 45 Han Chinese and 45 Japanese from Tokyo). Data was initially segmented across all chromosomes and a reduced matrix (1465 CNV blocks) was obtained with varSimil $=0.99$ and subVariation $=0.90$. In Figure 6 we plot the subject scores in the principal axes subspace of a MCA. Labelling each individual with its corresponding group color, we observe that three populations, namely CEU, YRI and CHB-JPT are clearly differentiated. The finer separation between CHB and JPT is not captured at with this unsupervised classification. When analyzing a CNV association study, this loadings can be used to correct for differences in ancestries, as Price et al. [15] have done for SNP data using PCA scores. 


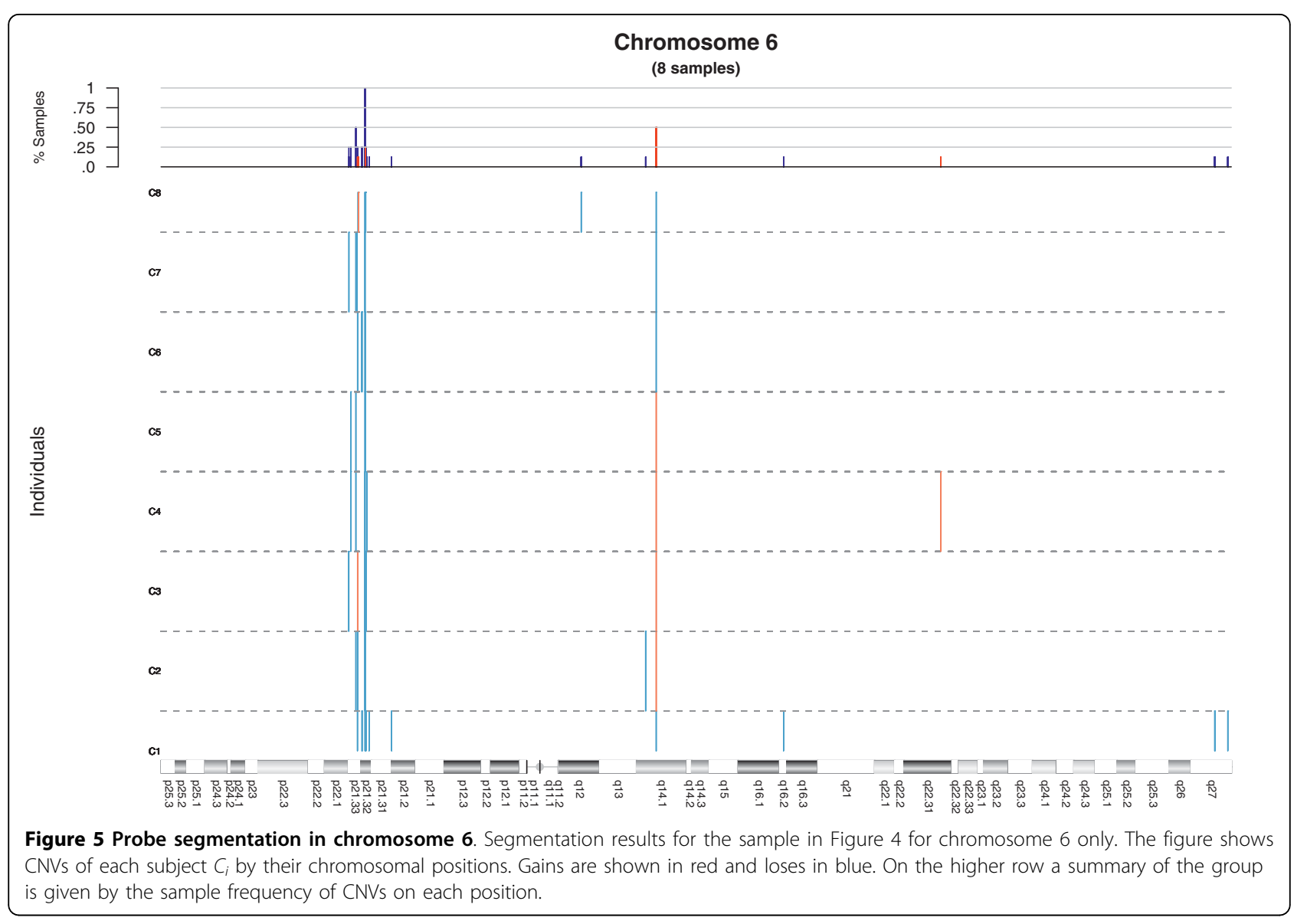

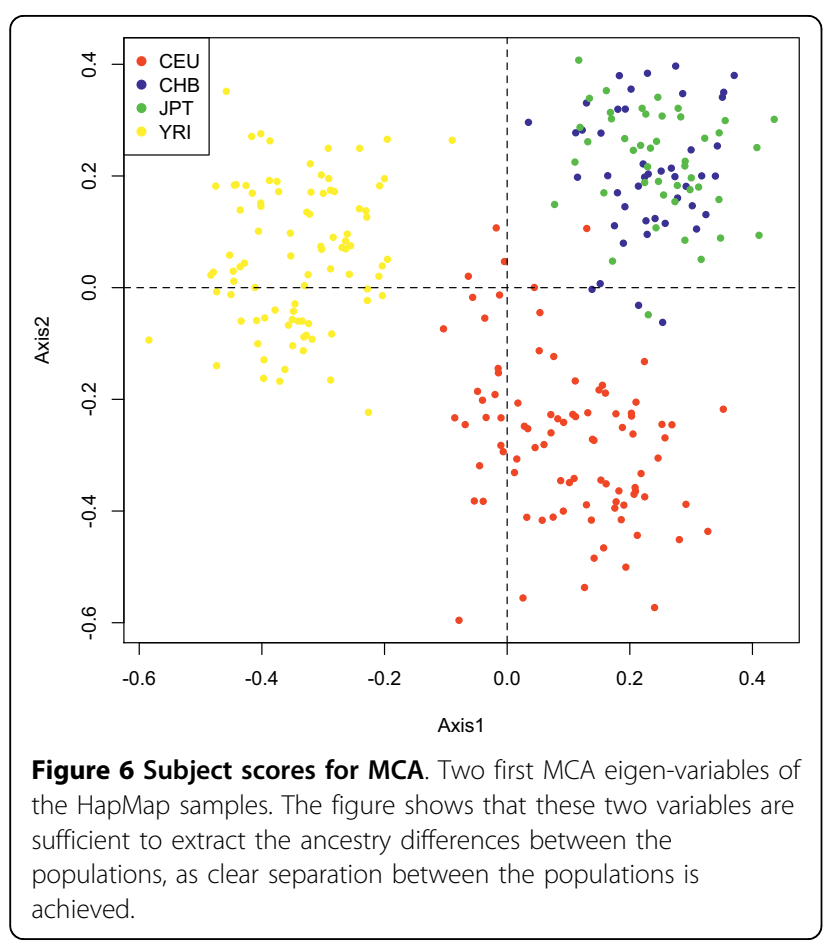

A supervised classification (MCDA) of the samples was performed with discrimin. cnv and followed by rank.variables. The ranking of the variables according to their maximum correlation across group axes is illustrated in Figure 7. We chose 87 variables, which had correlations higher than 0.5, to run an MCA. The results are shown in Figure 8. We observe that this reduced set of variables is able to classify, unsupervised, the populations similarly to the complete set of CNVs. This suggests that population ancestry can be accounted to a high degree with the sampling of very few markers. In addition, we run MCA for the two groups CHB-JPT and chose the optimal variables that revealed a degree of separation between such groups, see Figure 9.

\section{Association analysis}

One of the main aims of CNVs studies is to assess association between CNVs and disease. In order to illustrate how to perform this analysis using gada, we have generated case-control data for HapMap samples. We have randomly generated cases and controls with higher proportion of cases for YRI population. Figure 10 is a 


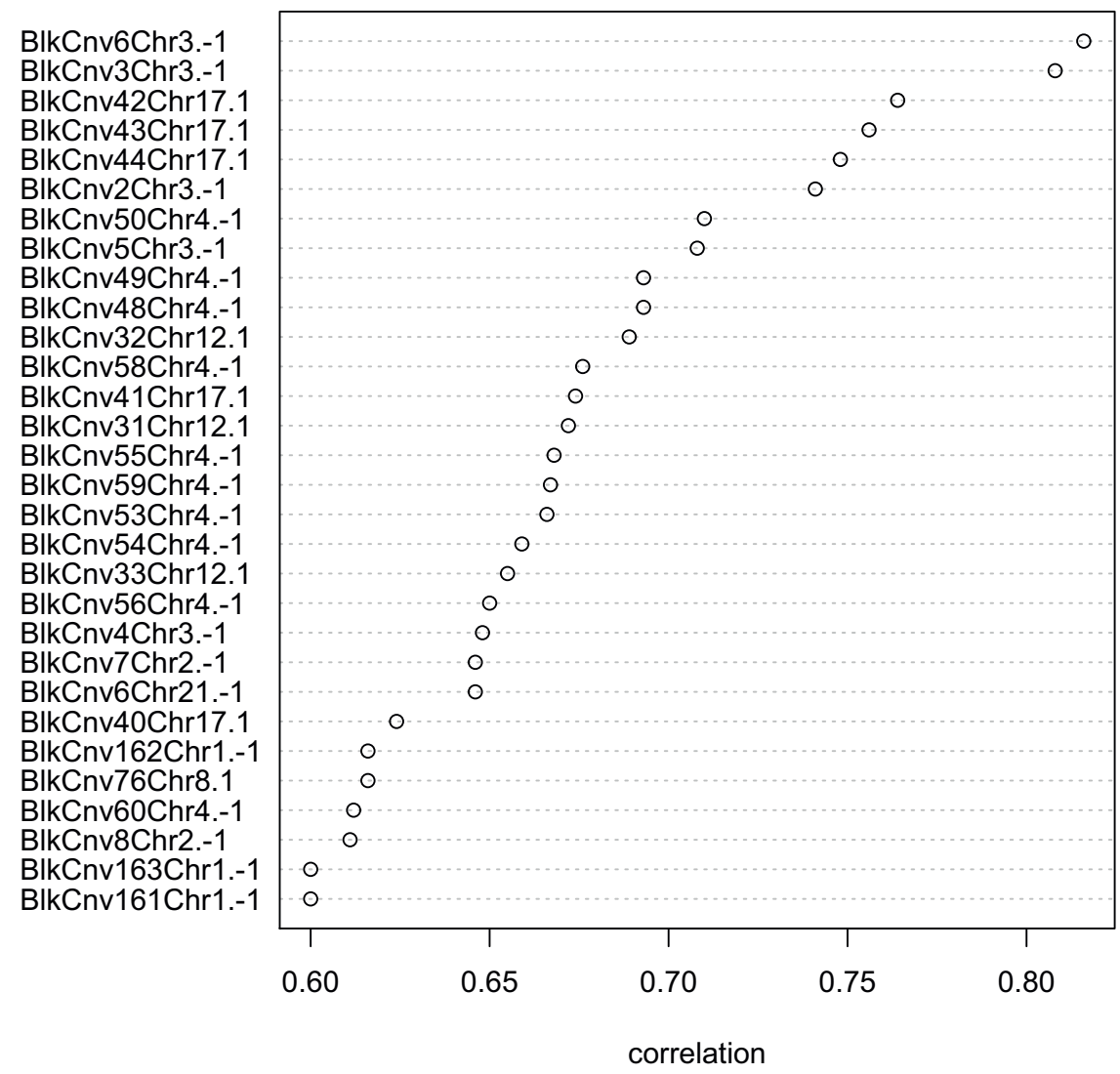

Figure 7 Variable ranking for MCDA. CNV blocks (recurrent CNVs) are ranked according to equation 4, that is their maximum correlation across class axes within the principal component subspace. Variables that are more relevant to the discrimination have higher correlations. The figure after the dot refers to relevant level (-1: loss, 0: no gain, 1:gain) of the CNV to discriminate one of the populations.

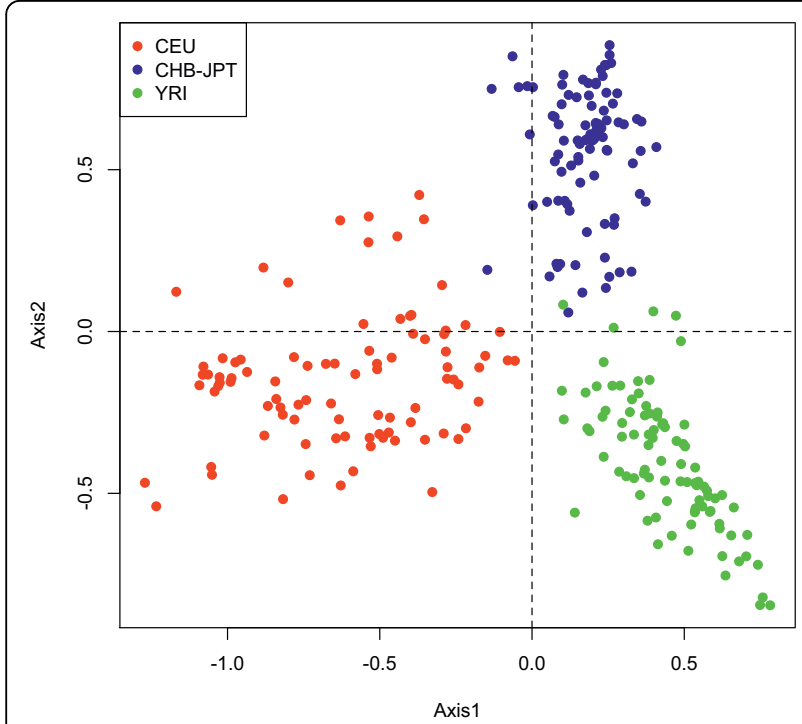

Figure 8 Subject scores for MCA. Multivariate analysis run with the highest 87 CNV blocks (corr > 0:5) ranked with MCDA. The figure shows a high degree of classification for such a reduced set, suggesting that ancestry can be fully accounted with a set of selected CNVs.

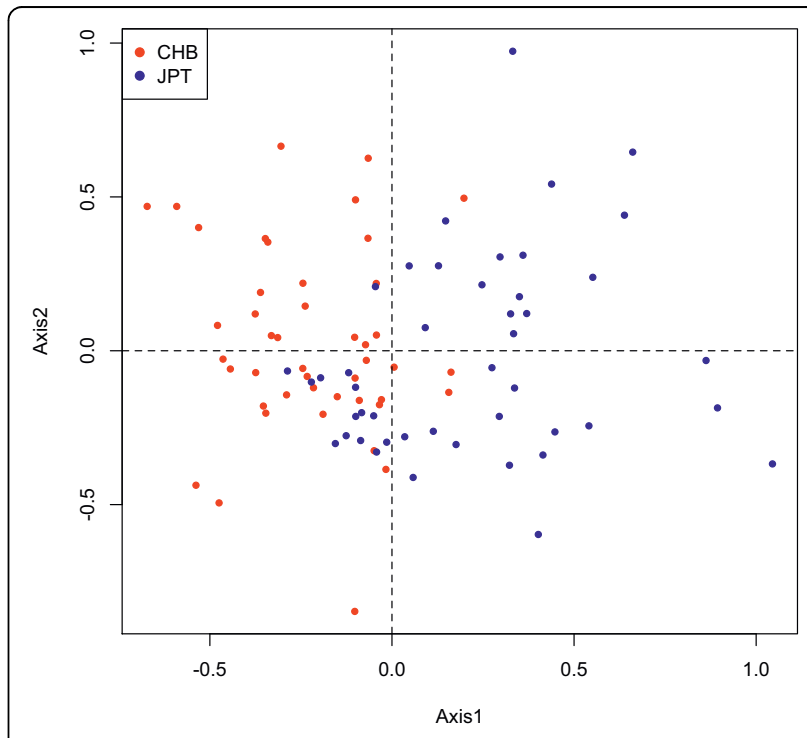

Figure 9 Subject scores for MCA. Multivariate analysis for optimal set of variables ranked with MCDA (corr > 0:5). The figure shows a degree of differentiation between the CHB and JPT populations. 


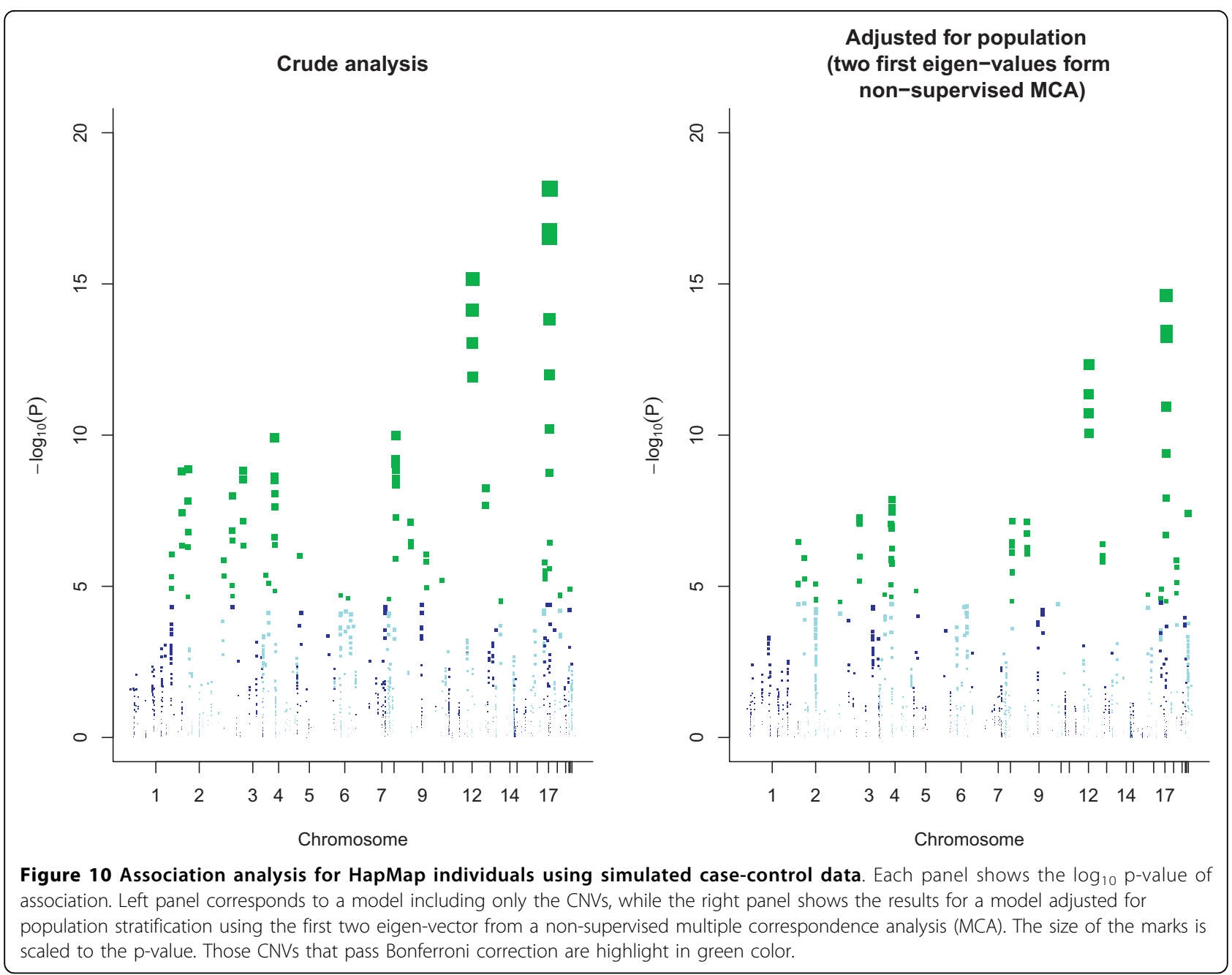

typical outcome of an association analysis. Here, we illustrate the $-\log _{10} p$ values for association analyses with and without adjusting for population stratification, showing the adequate correction achieved by using MCA.

\section{Conclusions}

We present a package that implements a very flexible pipeline for coupling diverse steps in the analysis of copy number alteration studies. The pipeline integrates in a single R-package the main components of such analysis, such as consecutively coupling normalization, segmentation, recurrent region identification, population stratification analysis and association tests. This unified frame-work and its implementation allow different modules to be easily substituted as new methods or improvements are made.

Some methods incorporate B-allele frequency in the detection of CNVs. Although GADA does not use this information, the algorithm is as precise as CBS and other algorithms that do use such information [8]. It has been shown, however, that the segmentation of the B-allele frequency is informative in detecting mosaicisms as described in [16]. The implementation of GADA in the present pipeline is easily adaptable to this type of analysis and is illustrated in http://groups.google.com/group/gadaproject.

Among its main features, the pipeline enables the import of data from multiple sources (e.g., Affymetrix GTC, Illumina and Aroma.affymetrix). In addition, its segmentation algorithm is very fast, easily parallelized, and its sensitivity can be adjusted quickly. Multiple plotting tools are offered to display results, which can also be exported back into standard formats or used in further R-based analyses. The R-GADA package also integrates new tools for identifying population structure and association analysis. The pipeline is applied to the HapMap samples, illustrating the importance of all the steps in the final association analysis. The package should be specially useful for upcoming CNV association studies which are expected to increase in the number of subjects and probes tested. The segmentation 
kernel is especially suited for detecting rare and small CNVs, but further efforts are required to develop novel tools that can link these CNVs to underlying functional elements on the DNA sequence.

\section{Availability and requirements}

- Project name: Gada Project

- Project home page: http://groups.google.com/ group/gadaproject

- Operating system(s): Platform independent

- Programming language: R 2.9.0

- License: GNU GPL

\section{Additional material}

Additional file 1: User's Manual. gada-manual.pdf is the user's guide of gada, where step-by-step segmentation on two sample data sets and the classification of the HapMap groups are described in detail.

\section{Acknowledgements}

RPR acknowledges helpful discussions with Prof. A. Ortega and Dr. S. Asgharzadeh who also provided support by grants K12-CA60104 from the $\mathrm{NIHs}$ Child Health Research Career Development Award Program. JRG wants to thank helpful comments and suggestions from Lluis Armengol and feedback from people from Xavier Estivill lab. The Genetic Unit from Universitat Pompeu Fabra is also acknowledged for providing Illumina $1 \mathrm{M}$ data. This work has been partially supported by the Spanish Ministry of Science and Innovation (MTM2008-02457 to JRG). We also acknowledge Dr. $\mathrm{H}$. Bengtsson for providing assistance in combining gada with aroma. affymetrix.

\section{Author details}

'Signal and Image Processing Institute, Viterbi School of Engineering, University of Southern California, EEB 400, 3740 McClintock Ave, Los Angeles, CA 90089-2564, USA. ${ }^{2}$ Division of Hematology - Oncology, Department of Pediatrics, Childrens Hospital Los Angeles, 4650 Sunset Boulevard Los Angeles, CA 90027, USA. ${ }^{3}$ Center for Research in Environmental Epidemiology (CREAL), Doctor Aiguader, 88, Barcelona, 08003, Spain. ${ }^{4}$ Institut Municipal d'Investigació Mèdica (IMIM), Doctor Aiguader, 88, Barcelona, 08003, Spain. ${ }^{5} \mathrm{CIBER}$ Epidemiología y Salud Pública (CIBERESP), Doctor Aiguader, 88, Barcelona, 08003, Spain.

\section{Authors' contributions}

RPR designed and improved GADA. JRG and RPR implemented the $R$ functions and wrote the article. JRG built the software package. AC implemented the multivariate analysis, analyzed HapMap data and revised the manuscript. All authors read and approved the final manuscript

Received: 17 December 2009 Accepted: 16 July 2010

Published: 16 July 2010

\section{References}

1. lafrate AJ, Feuk L, Rivera MN, Listewnik ML, Donahoe PK, Qi Y, Scherer SW, Lee C: Detection of large-scale variation in the human genome. Nat Genet 2004, 36(9):949-51.

2. Redon R, Ishikawa S, Fitch KR, Feuk L, Perry GH, Andrews TD, Fiegler $H$, Shapero MH, Carson AR, Chen W, Cho EK, Dallaire S, Freeman JL, Gonzalez JR, Gratacos M, et al: Global variation in copy number in the human genome. Nature 2006, 444(7118):444-54.

3. Kidd JM, Cooper GM, Donahue WF, Hayden HS, Sampas N, Graves T, Hansen N, Teague B, Alkan C, Antonacci F, Haugen E, Zerr T, Yamada NA, Tsang P, Newman TL, Tuzun E, Cheng Z, Ebling HM, Tusneem N, David R, Gillett W, Phelps KA, Weaver M, Saranga D, Brand A, Tao W, Gustafson E, McKernan K, Chen L, Malig M, Smith JD, Korn JM, McCarroll SA,
Altshuler DA, Peiffer DA, Dorschner M, Stamatoyannopoulos J, Schwartz D, Nickerson DA, Mullikin JC, Wilson RK, Bruhn L, Olson MV, Kaul R, Smith DR, Eichler EE: Mapping and sequencing of structural variation from eight human genomes. Nature 2008, 453(7191):56-64.

4. McCarroll S, Kuruvilla F, Korn J, Cawley S, Nemesh J, Wysoker A, Shapero M, de Bakker P, Maller J, Kirby A, Elliott A, Parkin M, Hubbell E, Webster T, Mei R, Veitch J, Collins P, Handsaker R, Lincoln S, Nizzari M, Blume J, Jones K, Rava R, Daly M, Gabriel S, Altshuler D: Integrated detection and population-genetic analysis of SNPs and copy number variation. Nat Genet 2008, 40(10):1166-74

5. Conrad DF, Pinto D, Redon R, Feuk L, Gokcumen O, Zhang Y, Aerts J, Andrews TD, Barnes C, Campbell P, Fitzgerald T, Hu M, Ihm CH, Kristiansson K, Macarthur DG, Macdonald JR, Onyiah I, Pang AWC, Robson S, Stirrups K, Valsesia A, Walter K, Wei J, Tyler-Smith C, Carter NP, Lee C, Scherer SW, Hurles ME: Origins and functional impact of copy number variation in the human genome. Nature 2010, 464(7289):704-712.

6. Manolio TA, Collins FS, Cox NJ, Goldstein DB, Hindorff LA, Hunter DJ, McCarthy MI, Ramos EM, Cardon LR, Chakravarti A, Cho JH, Guttmacher AE, Kong A, Kruglyak L, Mardis E, Rotimi CN, Slatkin M, Valle D, Whittemore AS, Boehnke M, Clark AG, Eichler EE, Gibson G, Haines JL, Mackay TFC, McCarroll SA, Visscher PM: Finding the missing heritability of complex diseases. Nature 2009, 461(7265):747-753.

7. Perry G, Ben-Dor A, Tsalenko A, Sampas N, Rodriguez-Revenga L, Tran C, Scheffer A, Steinfeld I, Tsang P, Yamada N, Park H, Kim J, Seo J, Yakhini Z, Laderman S, Bruhn L, Lee C: The fine-scale and complex architecture of human copy-number variation. Am J Hum Genet 2008, 82(3):685-95.

8. Pique-Regi R, Monso-Varona J, Ortega A, Seeger RC, Triche TJ, Asgharzadeh S: Sparse representation and Bayesian detection of genome copy number alterations from microarray data. Bioinformatics 2008, 24(3):309-18.

9. Olshen $A B$, Venkatraman $E S$, Lucito $R$, Wigler M: Circular binary segmentation for the analysis of array-based DNA copy number data. Biostatistics 2004, 5(4):557-72.

10. Winchester L, Yau C, Ragoussis J: Comparing CNV detection methods for SNP arrays. Brief Funct Genomic Proteomic 2009, 8(5):353-366.

11. Tipping ME: Sparse Bayesian learning and the relevance vector machine. J Mach Learn Res 2001, 1:211-244.

12. Greenacre MJ: Theory and applications of correspondence analysis Accademic Press, London 1984.

13. Caceres A, Basagaña X, Gonzalez JR: Multiple correspondence discriminant analysis: An application to detect stratification in copy number variation. Statistics in Medicine 2010, [Accepted February 2010].

14. Willenbrock $H$, Fridlyand J: A comparison study: applying segmentation to array CGH data for downstram analyses. Bioinformatics 2005, 21:4084-4091.

15. Price AL, Patterson NJ, Plenge RM, Weinblatt ME, Shadick NA, Reich D: Principal components analysis corrects for stratification in genome-wide association studies. Nat Genet 2006, 38(8):904-909.

16. Wang K, Li M, Hadley D, Liu R, Glessner J, Grant S, Hakonarson H, M B PennCNV: an integrated hidden Markov model designed for highresolution copy number variation detection in whole-genome SNP genotyping data. Genome Research 2007, 17:1665-1674.

doi:10.1186/1471-2105-11-380

Cite this article as: Pique-Regi et al.: R-Gada: a fast and flexible pipeline for copy number analysis in association studies. BMC Bioinformatics 2010 11:380. 